\title{
Infrared spectral profiles in liquids and atom-diatom interactions
}

\author{
A. Medina, ${ }^{\text {a) }}$ J. M. M. Roco, A. Calvo Hernández, and S. Velasco \\ Departamento de Física Aplicada, Facultad de Ciencias, Universidad de Salamanca, \\ 37008 Salamanca, Spain
}

(Received 8 June 2004; accepted 14 July 2004)

\begin{abstract}
Molecular dynamics simulations of the infrared spectrum of a generic simple polar diatomic in a liquid nonpolar solvent allow to reproduce the different prototypical experimental line shapes of this kind of systems. This is feasible by using different solute-solvent anisotropic potentials at fixed thermodynamic conditions. In the limit cases, the rotation of the diatomic is explained in terms of a quasifree motion or a rotational diffusion evolution and the spectra show a doublet structure formed by $P$ and $R$ branches or a unique collapsed branch, respectively. When the profile contains three branches, including an intense $Q$ branch in the vicinity of the center of the band, rotational evolution presents a particular hindering that can be understood by studying the influence on rotational spectral densities of the different time scales involved in rotational relaxation. Cancellation/enhancement effects among spectral density terms arising from intermediate and long times $(0.4-1 \mathrm{ps})$ are essential to understand rotational hindering. (C) 2004 American Institute of Physics. [DOI: 10.1063/1.1789937]
\end{abstract}

\section{INTRODUCTION}

Theoretical and experimental research on the spectroscopy of molecules in dense phases gives basic information on intermolecular interactions. ${ }^{1}$ All spectral features as position, intensity, or line shape are determined by the external and internal degrees of freedom of the molecular probe, and change because of its interactions with the surrounding solvent. ${ }^{2}$ In particular, polar diatomic molecules highly diluted in nonpolar fluids constitute the simplest manageable problem, thus allowing detailed theoretical analysis. Comparison with experimental data provides valuable information on the characteristics of microscopic interactions. Nevertheless some particular aspects, specially the theoretical interpretation of the shape of the bands for certain systems (e.g., hydrogen halides in spherical dense solvents) do still constitute an open question. ${ }^{3}$

Rotation-vibration spectra in the near infrared are easier to obtain than, for instance, in the far infrared, thus experimental data on the rovibrational fundamental bands of diatomics in dense fluids are relatively abundant. In rarefied gases these bands consist of individual rotational lines distributed in two branches, so called $P$ and $R$ branches, which form a doublet around the frequencies of the pure vibrational transitions. These bands are perturbed with increasing solvent density and, at liquid/dense fluid conditions, bands of most systems lack of fine rotational structure (or maintain only localized traces of fine structure on the band wings). Prototypical spectral profiles could be divided in three types: doublets formed by $P$ and $R$ branches with a more or less pronounced dip in between, triplets with a third branch $(Q$ branch) located near the band center that is dipole forbidden

\footnotetext{
a) Author to whom correspondence should be addressed; Electronic mail: amd385@usal.es
}

for isolated species, and a unique asymmetric branch as a result of spectral collapse.

Although spectral doublet-shaped bands are typical of gas solvents where the molecular probe executes a quasifree rotation, they have been also found, for instance, in the case of $\mathrm{CO}$ solved in $\mathrm{Ar}$ or Xe at liquid and dense gas conditions, $\mathrm{HCl}$ and $\mathrm{DCl}$ solved in liquid $\mathrm{SF}_{6} \cdot{ }^{2,4}$ In these cases, individual absorption lines are broad enough, hence the band displays only remainders of rotational structure at high $j$ values. In the case of $\mathrm{CO}$ solved in noble gases ${ }^{5,6}$ at moderately high liquid densities the spectra are associated to rotational diffusion of the molecular orientation. The band shows a single $Q$ branch, which progressively narrows as density increases. ${ }^{4}$ Between these two outermost situations some systems show a band with three visible maxima, where sometimes the central $Q$ branch is more intense than the $R$ branch and in other occasions the $Q$-branch peak is masked by interference effects and the spectrum intensity between $P$ and $R$ branches shows an almost monotonic behavior. ${ }^{3}$ These systems are essentially hydrogen halides (or isotopic derivations) solved in liquified noble gases ${ }^{7-14}$ or their mixtures, ${ }^{3}$ but also solved in isotropic solvents such as $\mathrm{CCl}_{4}$ or $\mathrm{SF}_{6} .{ }^{15}$

From a theoretical viewpoint there are precise formal theories (that in most occasions consider a quantum free rigid rotor as a starting point) capable to predict the broadening of rotational lines and their interferences from the gas phase to the liquid. ${ }^{4,16}$ Nevertheless, they fail in the description of the particular rotational motion that yields a triplet structure of the bands with $Q$-branch intensity comparable to that of the other spectral branches. Only phenomenological descriptions in which it is supposed that the simultaneous existence of molecules executing either quasifree rotations or rotational diffusion have been proposed. ${ }^{17}$ Interesting efforts have been recently devoted to characterize the hindered rotation that seems to be at the origin of $Q$-branch absorption. ${ }^{15}$ However, a full theoretical description of this problem is still 
a challenging question to solve in the spectroscopy of dense phases.

From long time ago molecular dynamics (MD) simulations have been shown to be a powerful tool to investigate the physical origin of infrared spectral band shapes of simple molecules in liquid solvents. ${ }^{18,19}$ Recently, it has been proposed that the hindering of rotational evolution of the diatomic in spectra having $Q$ branch is associated to the formation of rotational pseudocomplexes arising from the projection to the liquid phase of the well-known van der Waals complexes that, for example, hydrogen halides form in the gas phase. ${ }^{3,20}$ These pseudocomplexes, e.g., $\mathrm{Ar}-\mathrm{H}-\mathrm{Cl}$, perform a hindered rotational motion due to its relatively large moment of inertia. Moreover, the central region of the spectral line shapes is very sensitive to the anisotropic solute-solvent potential and thus provides an outstanding test to translate anisotropic interactions to peculiarities of rotational evolution. ${ }^{3,21}$ The objective of this work is to reproduce the prototypical experimental lineshapes by means of MD simulations where different atom-diatom anisotropic potentials are considered for a test system. The interpretation of the rotational motion of the molecule as obtained from each potential gives us a direct classical explanation of the physical basis of the spectral line shapes.

The paper is organized as follows: in Sec. II we briefly summarize the details of the simulations and the chosen potential surfaces. The recovering of experimental line shapes will allow us to conclude that a generic system in fixed liquid conditions can present any of the typical spectral line shapes by simply modifying the solute-solvent interaction. In Sec. III we analyze the orientational time correlation functions and the spectral densities associated to each potential in order to understand the different kinds of rotational evolutions that they yield. In Sec. IV we perform a detailed study of spectral densities by making a decomposition in terms of the different contributions arising from the diverse time scales present in the time orientational evolution of the diatomic. Finally, from Sec. V we conclude by exposing a classical image of how the rotation of the diatomic is directly reflected in the shape of spectral bands.

\section{RECOVERING THE PROTOTYPICAL EXPERIMENTAL LINE SHAPES}

\section{A. Theoretical background}

We make use of linear response theory to express the infrared absorption coefficient $\alpha(\omega)$ in terms of the classical spectral density $I(\omega)$,

$$
\alpha(\omega) \simeq \frac{\beta \hbar \omega}{2} \sinh \left(\frac{\beta \hbar \omega}{2}\right) I(\omega),
$$

where the population factor was proposed by Berens and Wilson $^{18}$ as a quantum correction to the classical pure rotational band contour. The spectral density is evaluated by means of classical MD simulations as the Fourier-Laplace transform of the infrared rotovibrational time correlation function $C_{R V}(t)$

$$
I(\omega)=\int_{0}^{\infty} \cos (\omega l) C_{R V}(l) d t,
$$

where we assume that the relaxation time scales associated to the vibration and the rotation of the diatomic are separable, i.e.,

$$
\begin{aligned}
C_{R V}(t)= & \langle[\Delta x(0) \vec{\mu}(0)] \cdot[\Delta x(t) \vec{\mu}(t)]\rangle \simeq\langle\Delta x(0) \Delta x(t)\rangle \\
& \times\langle\vec{\mu}(0) \cdot \vec{\mu}(t)\rangle \equiv C_{V}(t) C_{R}(t)
\end{aligned}
$$

being $\Delta x(t)$ the instantaneous separation of the atoms constituting the diatom from its equilibrium distance and $\vec{\mu}(t)$ is the permanent dipole moment of the diatomic. By obtaining separately $C_{R}(t)$ and $C_{V}(t)$, and using the convolution theorem it is possible to individually calculate the spectral densities associated to vibrational and rotational degrees of freedom as

$$
\alpha(\omega) \simeq \frac{\beta \hbar \omega}{2} \sinh \left(\frac{\beta \hbar \omega}{2}\right)\left[I_{V}(\omega) * I_{R}(\omega)\right],
$$

where

$$
\begin{aligned}
& I_{V}(\omega)=\int_{0}^{\infty} \cos (\omega t) C_{V}(t) d t, \\
& I_{R}(\omega)=\int_{0}^{\infty} \cos (\omega t) C_{R}(t) d t .
\end{aligned}
$$

\section{B. Molecular dynamics simulations}

We used the molecular dynamics software developed by our group in the last few years. Validation guidelines proposed by van Gunsteren and Mark $^{22}$ have been considered. In previous works ${ }^{3,20}$ our simulations were able to reproduce triplet-structured experimental infrared bands of nonpolar diatomic molecules solved in liquid noble gases and their mixtures at several liquid conditions. Moreover, the mentioned works showed the sensitivity of infrared lineshapes to atomdiatom anisotropic interactions in the case of highly anisotropic systems like $\mathrm{HCl}-\mathrm{Ar}$.

We considered a single diatom inside a sample of 255 solvent atoms interacting via a 12-6 Lennard-Jones potential. Although we were not specially interested in the peculiarities of the solvent, we considered Lennard-Jones parameters for pure Ar, $\epsilon_{\mathrm{Ar}-\mathrm{Ar}}=119.8 \mathrm{~K}$ and $\sigma_{\mathrm{Ar}-\mathrm{Ar}}=3.405 \AA$. We refer to the following section for details on solute-solvent interactions. Lorentz-Berthelot combination rules were applied between unlike atoms. Equations of motion were integrated through a leapfrog Verlet algorithm and usual periodic boundary conditions were considered. Simulations were divided in five blocks of $2 \times 10^{6} \Delta t$ with a time step small enough to correctly reproduce the vibration of the diatomic, $\Delta t=0.5 \times 10^{-3} \mathrm{ps}$. The total duration of the runs was elected to grant good statistics for the calculation of the seeked time correlation functions. Following the criteria proposed by Frenke and Smit, ${ }^{23}$ relative errors on these functions either at short or long times do not exceed $10^{-4}$. Equilibration period (100 ps) was also much longer than the expected decays of time correlation functions $(\sim 1 \mathrm{ps})$. In all cases thermodynamic conditions for the solvent were temperature $T$ 
TABLE I. Lennard-Jones site-site parameters of the anisotropic atomdiatom potentials used to obtain simulated infrared profiles.

\begin{tabular}{|c|c|c|c|}
\hline \multicolumn{4}{|c|}{$\epsilon(\mathrm{K})$} \\
\hline & SSI & SSF2 & SSP \\
\hline $\mathrm{H}-\mathrm{Ar}$ & 1.4 & 79.5 & 40.07 \\
\hline $\mathrm{Cl}-\mathrm{Ar}$ & 171.0 & 150.3 & 144.2 \\
\hline \multicolumn{4}{|c|}{$\sigma(\AA)$} \\
\hline $\mathrm{H}-\mathrm{Ar}$ & 2.77 & 2.41 & 3.07 \\
\hline $\mathrm{Cl}-\mathrm{Ar}$ & 3.42 & 3.44 & 3.38 \\
\hline
\end{tabular}

$=115 \mathrm{~K}$ and density $\rho=1.20 \mathrm{~g} / \mathrm{cm}^{3}$, i.e., a liquid state for $\mathrm{Ar}$ below its critical temperature. The vibration of the diatomic was represented through a Morse potential. We took a set of realistic parameters ${ }^{24}$ e.g., for isolated $\mathrm{HCl}$ : equilibrium distance $1.274 \AA$, dissociation energy $4.29 \times 10^{4} \mathrm{~cm}^{-1}$, and exponential parameter $\alpha=1.739 \AA^{-1}$. Finally, we should point out that statistical errors of pseudocomplexes sizes (estimated by performing simulations in blocks) mentioned hereafter amount to $5 \times 10^{-3} \AA$ at the most.

\section{Site-site anisotropic interactions}

We have considered three different orientationdependent atom-diatom potentials $V(r, \theta)$ built up as 12-6 Lennard-Jones site-site interactions. ${ }^{3}$ Sites are located at the atomic positions of the diatom. We shall consider these potentials as prototypical anisotropic interactions without making reference to a particular system, although all of them were originally developed for a specially interesting pair $\mathrm{HCl}$-Ar. Parameters of the fits are presented in Table I, and contour diagrams of the three potential functions are displayed in Fig. 1.

The first potential considered shall be denoted SSI and is a site-site fit to the isotropic part of surface HWK II, developed by Holmgren et $a .^{25}$ This potential is an isotropic limit that obviously does not reproduce privileged presence of solvent atoms at any orientation [Fig. 1(a)].

The second potential that we shall consider (SSF2) was originally obtained as a site-site fit to the fully anisotropic potential HWK II. ${ }^{3,25}$ Its main advantage is that it reproduces the existence of $\mathrm{Ar}-\mathrm{H}-\mathrm{Cl}$ complexes at low gas densities by incorporating an absolute minimum at such linear orientation [see Fig. 1(b)]. This potential recently succeeded to reproduce the experimentally observed $Q$ branch in the infrared spectra of $\mathrm{HCl}$ solved in liquid Ar solutions. ${ }^{3}$ Moreover, it has been also shown that this potential retains in the liquid phase the privileged presence of Ar atoms at an approximately linear orientation with respect to $\mathrm{HCl}^{20}$ Concretely, at the liquid conditions we consider, it is $22 \%$ more probable to find an Ar atom at nearly linear orientation with respect to the internuclear axis than the average over all orientations.

Finally, we shall also employ a standard MD site-site potential constructed from atom-atom $(\mathrm{H}-\mathrm{H}$ and $\mathrm{Cl}-\mathrm{Cl})$ interactions combined through Lorentz-Berthelot rules (we shall denote it as SSP). As Fig. 1(c) shows, this potential incorporates an absolute minimum at around $\theta=60^{\circ}$ respect to the internuclear axis (we take $\theta=0$ as the linear $\mathrm{Ar}-\mathrm{H}-\mathrm{Cl}$ con-

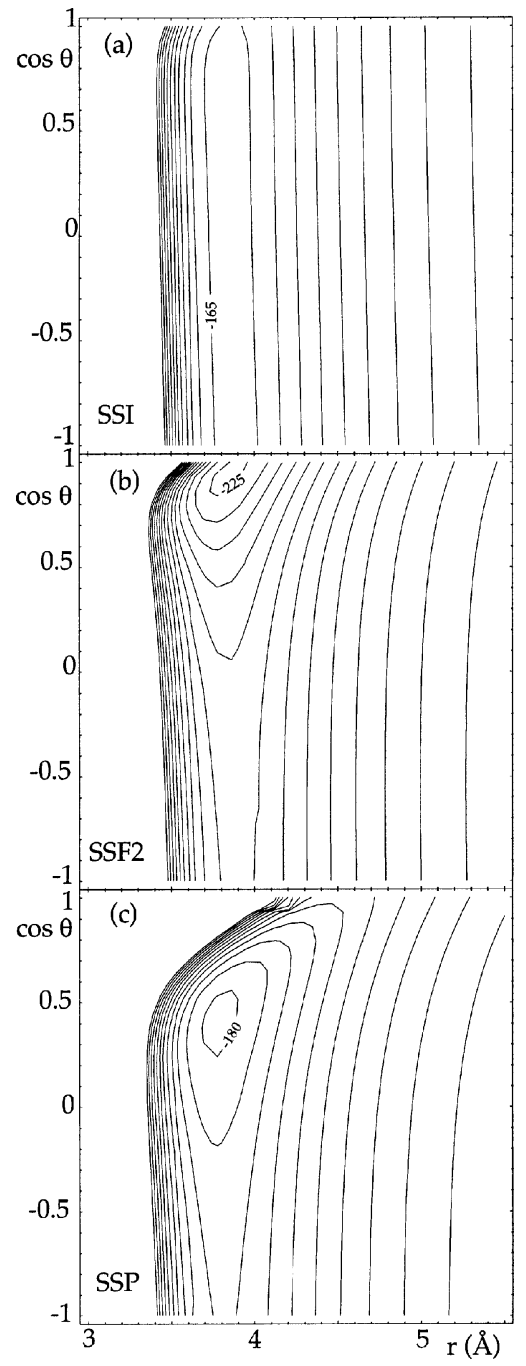

FIG. 1. Contour diagrams of the site-site anisotropic solute-solvent potentials, $V(r, \theta)(\mathrm{K})$ used in MD simulations. Contour intervals correspond to $\Delta V=15 \mathrm{~K}$. In the case of $\mathrm{HCl}-\mathrm{Ar}, \theta=0$ is associated to the linear configuration $\mathrm{Ar}-\mathrm{H}-\mathrm{Cl}$.

figuration). The corresponding well depth is 0.8 times that of SSF2. Another important difference with respect to SSF2 arises in the angular shape of the potential barrier at $\theta \simeq 0$ that is considerably apart from $\mathrm{H}$ position. SSP was implemented by Padró and Guàrdia ${ }^{26}$ in order to reproduce via MD simulations the infrared spectra of $\mathrm{HCl}$ in $\mathrm{Ar}$ at different liquid conditions. Global width of the bands were adequately achieved. With the same kind of calculations that we introduced in Ref. 20, for this potential it is obtained an excess presence of solvent atoms at linear situation around $31 \%$, i.e., in spite of its shallower well at quasilinear orientations, it also keeps in the liquid phase a privileged presence of solvent atoms at such location (in fact it predicts a $30 \%$ larger occurrence than SSF2).

\section{Simulated spectra}

The simulated spectra are displayed in Fig. 2. The one obtained from a site-site fit to an isotropic potential SSI appears as a classical band line shape with well separated $P$ and $R$ branches [Fig. 2(a)]. The relative intensity of the maxi- 


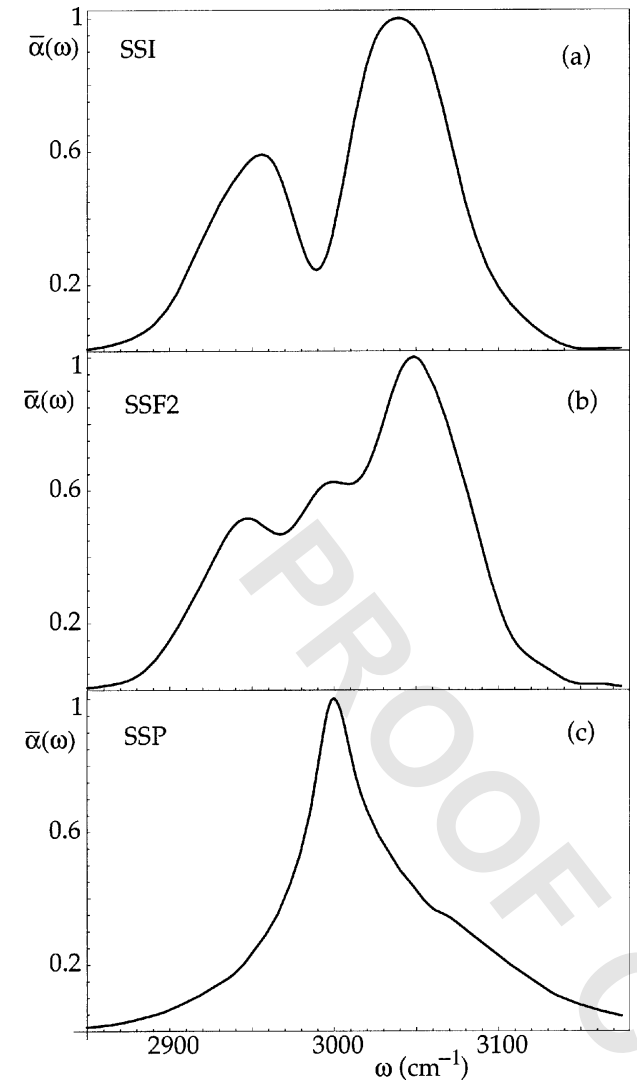

FIG. 2. Maximum intensity normalized spectral line shapes $\bar{\alpha}(\omega)$ obtained via MD simulations with the considered anisotropic interactions. Wave numbers should only be considered as a guide since solvent-induced shifts in the fundamental frequency of the diatomic were not considered.

mum band absorption peaks is 1:0.6. The distance between branches amounts $\approx 90 \mathrm{~cm}^{-1}$ and the interbranch region shows an absorption well with a minimum absorption around $75 \%$ less than the maximum one, the peak of the $R$ branch. With respect to the band position it is worth mentioning that we did not try to take into account the solvent-induced vibrational frequency shift because we do not compare directly with experimental bands, and thus our result corresponds to the fundamental vibrational frequency of the isolated rotator. A previous work ${ }^{3}$ showed that a band displacement of around $100 \mathrm{~cm}^{-1}$ should be applied in order to match experimental band positions. As mentioned in the Introduction such a line shape is experimentally visualized in systems like CO-Ar at dense gas conditions for the solvent, $\mathrm{CO}$ in liquid $\mathrm{Xe}$, and $\mathrm{DCl}$ in liquid $\mathrm{SF}_{6}$.

On the other side, the band contour obtained with the anisotropic potential surface SSP presents a single asymmetric peak due to the rotational structure collapse [Fig. 2(c)]. This is the case of a linear molecule such as $\mathrm{CO}$ for which $P$ and $R$ branches become a single one in the liquid phase at sufficiently high density. ${ }^{4}$ The collapsed branch is progressively narrowed as density increases. This collapse is the well-known spectral manifestation of the transition from free rotation of the molecule to rotational diffusion.

A third which as last but not least interesting case is the contour band obtained with anisotropic surface SSF2 [see Fig. 2(b)]. The classical line shape shows a triplet structure

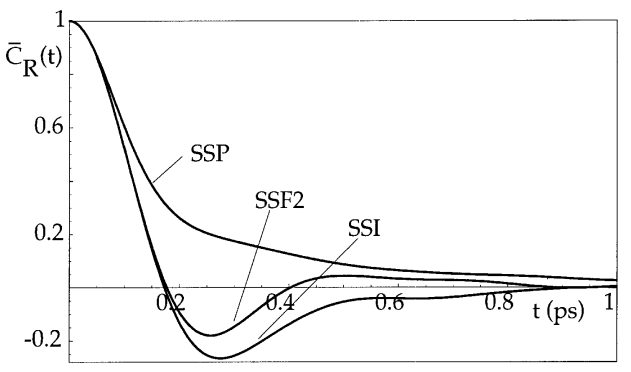

FIG. 3. Normalized orientational time correlation functions $\bar{C}_{R}(t)$ calculated from simulations.

with separated $P$ and $R$ branches (around $100 \mathrm{~cm}^{-1}$ ) as in the case SSI. But in the interbranch region does arise an intense $Q$ branch which is forbidden for the isolated diatomic. The relative intensity of the $Q$ branch amounts $63 \%$ respect to the absolute maximum. These particular line shapes have been experimentally reported for hydrogen halides such as $\mathrm{HBr}$, $\mathrm{HCl}$, and $\mathrm{HF}$ solved in liquid noble gases or larger spherical molecules as $\mathrm{SF}_{6}$ or $\mathrm{CCl}_{4}$. Actually, the spectrum shown in the picture correctly reproduces the experimental band of $\mathrm{HCl}$ in liquid $\mathrm{Ar}$ at $T=115 \mathrm{~K}$ as previously reported. ${ }^{3}$ This band was obtained on the basis of the anisotropic interaction SSF2 that, as commented in the preceding section, corresponds to a site-site fitting of a realistic potential that presents a deep minimum for the almost linear $\mathrm{Ar}-\mathrm{H}-\mathrm{Cl}$ arrangement.

In summary, by choosing particular solute-solvent interactions for a fixed solute-solvent pair and certain liquid conditions it is possible to reproduce the prototypical lineshapes revealed by experimental studies. This fact allow us to state that infrared profiles constitute a direct (and thus attracting) experimental macroscopic manifestation of microscopic anisotropic solute-solvent interactions (orientational relaxation).

\section{TIME AND FREQUENCY DOMAIN STUDIES OF REORIENTATION}

Our aim in this section is to deepen in the physics of the relationship among anisotropic interactions and reorientation of the linear rotor in the liquid by looking both at the orientational correlation functions in the time domain and at the spectral densities in the frequency domain.

Figure 3 shows the normalized time autocorrelation functions, $\bar{C}_{R}(t) \equiv C_{R}(t) / C_{R}(0)$, for the three potentials considered. Molecular reorientation corresponding to the doublet spectral structure obtained with an isotropic potential (SSI) displays a rapid decay at times lower than $0.2 \mathrm{ps}$ and reaches a deep negative minimum around $0.3 \mathrm{ps}$. This means that, to a considerable extent, the molecule achieves to rotate more than $90^{\circ}$ respect to its initial orientation. Afterwards, rotation relaxes as a monotonic exponential to complete decorrelation that is fulfilled around $1 \mathrm{ps}$. Moreover, this simulated correlation function is almost indistinguishable from that of a classical free rotor. ${ }^{20,27}$ Thus, it is clear that the isotropic potential yields a quasifree rotational relaxation.

The reorientational time correlation function associated to SSP can also be recovered in theoretical terms, just by 
appealing to a classical rotational diffusion model. ${ }^{4}$ Dipole moment relaxes to equilibrium through a roughly exponential decay that reflects, in terms of theoretical models, a sequence of short free rotations interrupted by collisions. Complete relaxation is reached after $\approx 2$ ps, i.e., long-time tails considerably increase the correlation time of this kind of reorientational evolution respect to the free case.

Dipole moment relaxation yielding a triplet spectral structure (potential SSF2) corresponds to a hindered rotation, as recently argued. ${ }^{20}$ The short time decay is analogous to a quasifree evolution, but after 0.2 ps differences are substantial. First, reorientation over $90^{\circ}$ is much less complete making the depth of the negative minimum smaller $(-0.25$ for SSI and -0.15 for SSF2). For times longer than 0.3 ps behavior of $\bar{C}_{R}(t)$ is now far from exponential. It takes again positive values in a evolution that can be considered as a damped oscillatory conduct. This means that the molecule turns its reorientation in inverse sense going back to angles below $90^{\circ}$ as it reaches a potential barrier. This is compatible with the existence of some kind of rotational species with larger moment of inertia than that of the isolated molecule, probably coming from the solute-solvent complexes SSF2 potential allows at low densities. In liquids, it has been used the term rotational pseudocomplex in order to shortly describe this kind of situation. Function $\bar{C}_{R}(t)$ goes to zero about 1 ps, i.e., in this case there is no evidence of long time tails like those appearing in rotational diffusion. This kind of evolution has not been reproduced up to date from formal spectral theories. It is directly related to the appearance of a $Q$ branch in the region between $P$ and $R$ branches as it has been shown in the last years from experimental ${ }^{13,28}$ and molecular dynamics simulations studies. ${ }^{3,20}$

It is illustrative at this point to compare for both anisotropic potentials the equilibrium size of pseudocomplexes with the shape of the solvent cage around the diatomic ${ }^{20}$ (if we can properly use the word cage in the liquid phase). This represents a structural measure of the degree of rotational hindering. For SSF2, the pseudocomplex size is $0.11 \AA$ larger than the minimum cage radius whereas for SSP it increases up to $0.26 \AA$. This result is coherent with the shape of the isopotential lines for SSP around $\theta=0$ shown in Fig. 1 and constitutes an alternative way to the study of $C_{R}(t)$ in order to understand the extent to which hindering is completed for different anisotropic interaction surfaces.

In the frequency domain, vibrorotational relaxation is visualized through the absorption coefficient $\alpha(\omega)$. As shown in Eq. (4), band profiles are given by the convolution of the vibrational and rotational spectral densities weighted up by the semiclassical population factor that gives the relative intensities of the branches forming the band. The vibrational spectral density $I_{V}(\omega)$ is a sharp $\delta$-like function located at the fundamental vibration frequency of the diatomic in the solution. When convoluting with the rotational part, it gives the position of the band center. The rotational spectral density $I_{R}(\omega)$ gives the shape of the band itself. On one hand, the existence of a single peak, a doublet, or a triplet structure is associated to the rotational evolution of the diatomic and, on the other hand to the existence of one or more relative maxima in $I_{R}(\omega)$. Figure 4 shows $I_{R}(\omega)$ for all the proto-

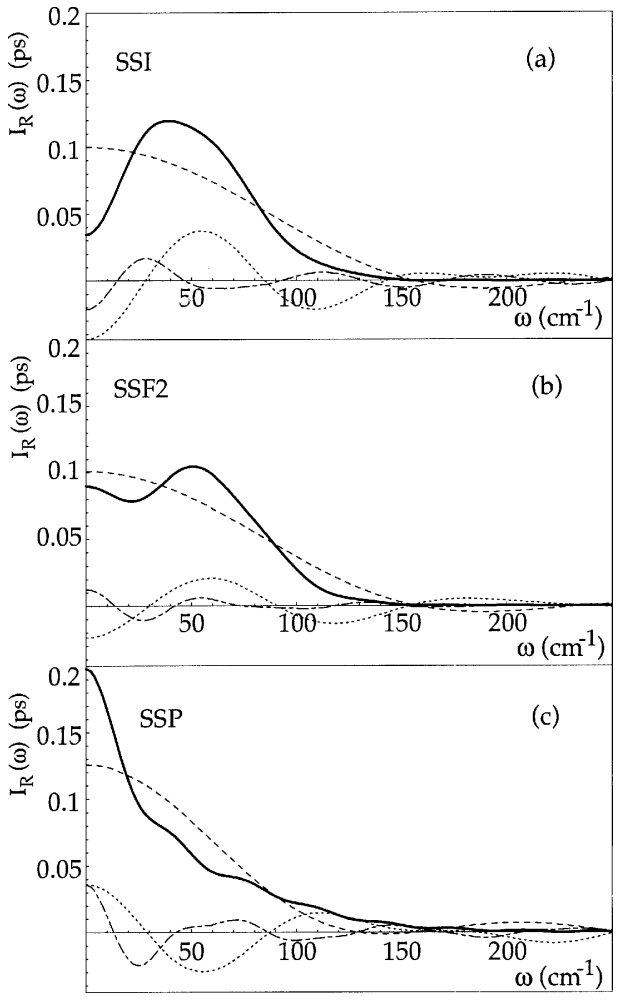

FIG. 4. Rotational spectral densities $I_{R}(\omega)$ (thick lines) obtained by Fourier transforming the orientational time correlation functions $C_{R}(t)$ and their reconstruction for the potential surfaces SSI, SSF2, and SSP by considering different time intervals in the integrations. Dashed line corresponds to the interval $[0,0.2] \mathrm{ps}\left[I_{R}^{(1)}(\omega)\right]$, dotted line to $[0.2,0.4]$ ps $\left[I_{R}^{(2)}(\omega)\right]$, and dashdotted line to $[0.4,1.0]$ ps $\left[I_{R}^{(3)}(\omega)\right]$.

typical band contours found in experimental studies, as obtained from our MD simulations with the considered site-site fitted potentials.

The isotropic potential SSI gives a rotational spectral density that only has one maximum at nonzero frequency and a deep well at $\omega=0$ [Fig. 4(a)]. Its convolution with $I_{V}(\omega)$ yields a doublet structure symmetric respect to the band center. After weighting up with the frequency dependent population factor the symmetry in the height of both maxima disappears and thus the absorption coefficient looks like a $P$ branch less intense than the $R$ branch. Moreover, there is a profound decrease of absorption in the interbranch region. Intensity in this zone directly comes from $I_{R}(\omega$ $=0)$, i.e., the integral correlation time of $C_{R}(t): t_{R}=I_{R}(\omega$ $=0)=\int_{0}^{\infty} C_{R}(t) d t$. In the case of SSI, we take the upper limit of the integrals as $1 \mathrm{ps}$, which gives $t_{R} \simeq 0.035 \mathrm{ps}$. Such a low value for $t_{R}$ is due to the existence in $C_{R}(t)$ of positive and negative areas that cancel each other. Therefore, the function goes to zero in a time $(\approx 1.0 \mathrm{ps})$ that is much larger than the integral correlation time.

On the other limit, simulations with SSP [Fig. 4(c)] yield a rotational spectral density with only one absolute maximum at zero frequency. This leads to a single peaked absorption coefficient, i.e., a collapsed band profile. Now $t_{R}$ is evaluated up to $2.0 \mathrm{ps}$ due to the long time tail of the corresponding $C_{R}(l)$ and gives a value of $0.20 \mathrm{ps}$, almost one order of magnitude larger than the SSI case.

The potential SSF2 gives a rich structure for $I_{R}(\omega)$ [Fig. 
4(b)], which displays two maxima and a smooth hollow between them. The absolute maximum (around $70 \mathrm{~cm}^{-1}$ ) corresponds to the $P$ or $R$ branches and the maximum at $\omega=0$ mirrors the $Q$ branch shown by $\alpha(\omega)$ in the spectral density. The intensity of the absolute maxima of $I_{R}(\omega)$ (slightly over $0.1 \mathrm{ps}$ ) is similar for SSI and SSF2, but, on the contrary, at zero frequency both curves take appreciably different values, $t_{R} \approx 0.035 \mathrm{ps}$ for SSI and $\approx 0.090 \mathrm{ps}$ for SSF2. This fact arises from the different cancellation effects among positive and negative areas in the corresponding $C_{R}(t)$. We shall come back over this interesting point later on.

As a general conclusion from the three potentials, we see that the rotational spectral density preserves the structure contained in $\alpha(\omega)$ and it serves as a bridge between the time evolution of the reorientation of the molecule and its spectral manifestation in the absorption coefficient. In the following section our interest will be centered on the translation to $I_{R}(\omega)$ of the particular details of the time evolution of $C_{R}(t)$ in different time intervals for each anisotropic potential.

\section{RECONSTRUCTING THE ROTATIONAL SPECTRAL DENSITIES}

The separation between vibrational and rotational degrees of freedom that we assumed in Eq. (4) allows for the direct study of the different time scales of reorientational relaxation. This can be made by evaluating $I_{R}(\omega)$ as a sum of contributions from different time intervals, i.e., $I_{R}(\omega)$ $=\Sigma_{i} I_{R}^{(i)}(\omega)$, where $I_{R}^{(i)}(\omega)=\int_{\alpha_{i}}^{b_{i}} \cos (\omega t) C_{R}(t) d t$. We have evaluated $I_{R}^{(i)}(\omega)$ in three different time intervals: the first rapid decay of $C_{R}(t),[0,0.2]$ (ps) [that we shall denote with the superscript $(1)]$, the intermediate region containing the negative well, $[0.2,0.4]$ (ps) [superscript (2)], and the relaxation before long time tails, $[0.4,1]$ (ps) [superscript (3)]. The different components of $I_{R}(\omega)$ are shown in Fig. 4.

The isotropic potential SSI leads to a rotational spectral density characterized by an absolute maximum around 30 $\mathrm{cm}^{-1}$ and a pronounced dip at zero frequency. The component associated to the rapid exponential decay of $C_{R}(t)$, $I_{R}^{(1)}(\omega)$ is essentially lorentzian with a zero value of $0.1 \mathrm{ps}$. The negative well of $C_{R}(t)$ yields a damped oscillatory component, $I_{R}^{(2)}(\omega)$, with large negative initial amplitude and an absolute maximum around $50 \mathrm{~cm}^{-1}$. The negative exponential tail after the minimum of $C_{R}(t)$ gives another damped oscillatory component $I_{R}^{(3)}(\omega)$ less intense than $I_{R}^{(2)}(\omega)$ but also with negative zero value. The addition of the negative initial values of $I_{R}^{(2)}(\omega)$ and $I_{R}^{(3)}(\omega)$ gives rise to the initial dip in $I_{R}(\omega)$ because it drastically decreases the lorentzian value at $\omega=0$. The absolute maximum in $I_{R}(\omega)$ is due to the constructive interference between $I_{R}^{(2)}(\omega)$ and $I_{R}^{(3)}(\omega)$.

The potential that yields a collapsed line shape SSP originates a lorentzian component, $I_{R}^{(1)}(\omega)$ similar to that of SSI [see Fig. 4(c)]. All the remaining components of $I_{R}(\omega)$ are amplitude decreasing oscillatory terms. But their value at $\omega=0$ is now always positive, leading to an increase of the amplitude of the lorentzian without any intensity dip. In other words there exist an intensity enhancement in $I_{R}(\omega)$ at low frequencies that leads to a unique branch in the absorption coefficient.
In the rotational spectral density obtained from SSF2 there are three interesting aspects arising from dephasing between $I_{R}^{(2)}(\omega)$ and $I_{R}^{(3)}(\omega)$ [see Fig. 4(b)]: First, there exist partial cancellation effects at $\omega=0$ as a consequence of the positive values of $C_{R}(t)$ between 0.4 and 0.8 ps. Second, cancellation becomes negative enhancement between 15 and $30 \mathrm{~cm}^{-1}$, and third, negative enhancement becomes positive around $50 \mathrm{~cm}^{-1}$. These three factors originate the particular shape of $I_{R}(\omega)$, two maxima with a shallow well between them. In particular, the zero frequency maximum, that is transformed into a $Q$ branch in $\alpha(\omega)$ arises from the mentioned cancellation effects that are absent in the other interaction potentials. In other words, the damped oscillatory behavior of $C_{R}(t)$ between 0.2 and 0.8 ps (particularly the positive rebound between 0.4 and $0.8 \mathrm{ps)}$ ) provokes peculiar cancellation/enhancement effects in the low-frequency region of the spectral density, which finally originate the appearance of a visible $Q$ branch in the absorption coefficient.

\section{CONCLUDING REMARKS}

We have shown that classical MD simulations for a fixed solute-solvent pair and liquid solvent conditions allow to appraise the sensitivity of infrared bands to the peculiarities of the solute-solvent pair potential. This means that the particular features of the pair anisotropic potential are not completely masked by the surrounding fluid and are even essential for understanding the infrared spectroscopy of the system, and thus its rotational relaxation. This is one of the reasons why these simple systems are interesting, their macroscopic measurable infrared spectra constitute a good test to get information on the anisotropic interactions at microscopic level.

The well-known fact that vibrational relaxation of the diatomic and its rotational evolution take place in separable time scales give us the possibility of a parallel and complementary study in terms of times [dipole moment time autocorrelation function $C_{R}(t)$ ] and/or frequencies [spectral density, $\left.I_{R}(\omega)\right]$. Infrared contours with $P$ and $R$ branches separated by a more or less deep well arise from a quasifree rotational evolution of the diatomic. In these cases the interaction potential is almost isotropic and $C_{R}(t)$ displays a deep minimum around $0.3 \mathrm{ps}$ after a rapid initial decay. Afterwards, it shows a quasiexponential negative evolution towards decorrelation. In a simple classical scheme [see Fig. 5(a)] interaction with solvent atoms surrounding the diatomic allows it to rotate an angle over $90^{\circ}$ within a short time interval and to go on its quasifree evolution. In this case spectral density is characterized by a large negative enhancement at low frequencies between intermediate- and long-time components that transform into a deep valley in the absorption coefficient.

When the anisotropic solute-solvent potential allows for the formation of rotational pseudocomplexes at liquid densities (as a trace of stable complexes in the gas phase), the effective rotation of the molecule and the adjacent solvent atom gives rise to a peculiar hindered rotation in the first solvation shell. Now the diatomic rotates up to $90^{\circ}$ with respect to its initial orientation [see Fig. 5(b)], but feels a po- 

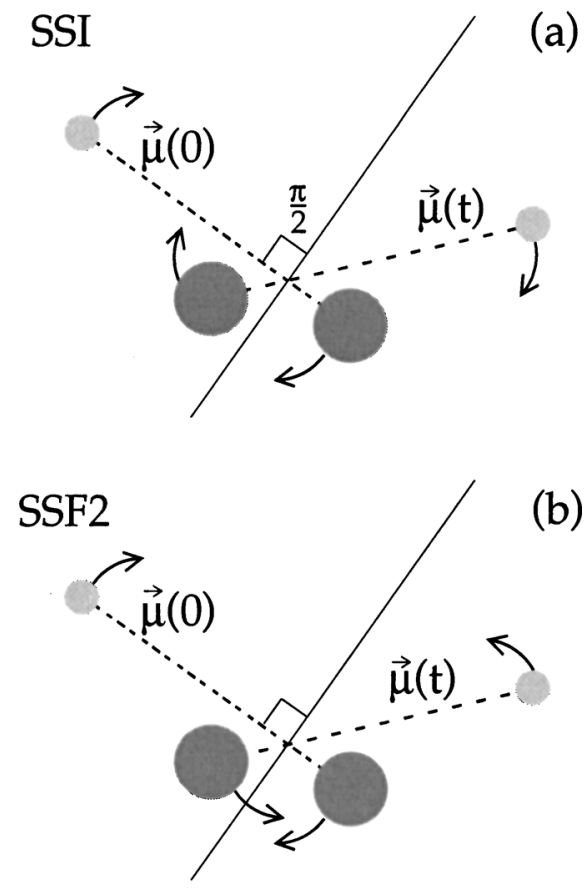

(b)

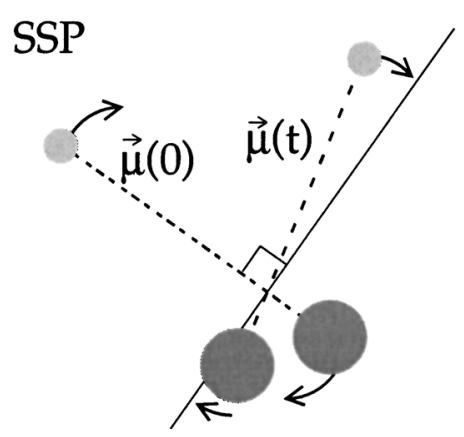

(c)

FIG. 5. Classical scheme of the time reorientation of the diatomic molecule in the liquid as obtained from the considered anisotropic molecule-solvent interactions. Scale does not necessarily corresponds to reality. Solid line represents a perpendicular axis respect to the initial orientation of the diatomic.

tential barrier that reverts rotation. After times around $0.4 \mathrm{ps}$ the orientation is again below $90^{\circ}$ with respect to initial conditions. This manifests in $C_{R}(t)$ as a negative shallow well between 0.2 and 0.4 ps and a subsequent damped oscillatory behavior that includes a positive bounce between 0.4 and 0.8 ps. This rotational relaxation is responsible for the existence of an intense $Q$ branch in the region between $P$ and $R$ branches that is forbidden at low densities. $Q$ branch comes from a balance between cancellation and enhancement effects in the components of the spectral density associated to each time scale in the rotational relaxation.

In the case where pseudocomplex rotational evolution is hindered to a great extent (because of the relative size of pseudocomplex and the space available for the molecule to rotate in the cage around) by the interactions with the surrounding solvent atoms, we can appeal to a rotational diffusion process, where the molecule is unable to revert its orientation in a short time [see Fig. 5(c)]. This yields a slow positive and monotonic exponential decay of $C_{R}(t)$ that spectrally corresponds to a collapsed band with a central in- tense $Q$ branch. In terms of spectral density, positive enhancement at low frequencies between all of the distinct time scales involved causes an absolute maximum at zero frequency and a monotonic intensity decrease for higher frequencies.

Finally, we would like to stress that the results obtained in this work are promising with respect to the finding of classical interpretations of other kind of spectral manifestations of solutes solved in simple liquid solvents. In this sense we would like to mention recent experimental results on the first vibrational overtone band of $\mathrm{HCl}$ in fluid Xe (Ref. 29) where a forbidden $Q$ branch is observed. Moreover, a rich structure has been found in the collision-induced absorption of $\mathrm{N}_{2}$ in $\mathrm{Ar}$ (Ref. 30) and $\mathrm{HD}$ in liquid $\mathrm{Ne},{ }^{31}$ the latter has been measured recently for the first time. On the other hand, this kind of classical studies of reorientation in liquids are required for future development of complete quantum theories incorporating rotational hindering.

\section{ACKNOWLEDGMENTS}

The authors thank financial support from Ministerio de Ciencia y Tecnología of Spain under Grant Nos. BFM200201225 FEDER and BFM2003-07106. The authors also acknowledge support by Junta de Castilla y León of Spain under Grant Nos. SA040/08 and SA092/04.

${ }^{1}$ M. O. Bulanin, N. D. Orlova, and G. Ya Zelikina, in Molecular Cryospectroscopy, edited by R. J. H. Clark and R. E. Hester (Wiley, Chichester, 1995), Chap. 3.

${ }^{2}$ M. O. Bulanin, S. Velasco, and A. Calvo Hernández, J. Mol. Liq. 70, 107 (1996).

${ }_{3}^{3}$ A. Medina, J. M. M. Roco, A. Calvo Hernández, S. Velasco, M. O. Bulanin, W. A. Herrebout, and B. J. van der Veken, J. Chem. Phys. 116, 5058 (2002).

${ }^{4}$ A. I. Burshtein and S. I. Temkin, Spectroscopy of Molecular Rotation in Gases and Liquids (Cambridge University Press, Cambridge, 1994).

${ }^{5}$ U. Buontempo, S. Cunsolo, and G. Jacucci, J. Chem. Phys. 59, 3750 (1973).

${ }^{6}$ N. N. Filippov and K. S. Rutkowski, J. Mol. Liq. 92, 251 (2001).

${ }^{7}$ J. Chesnoy, Chem. Phys. Lett. 114, 220 (1985).

${ }^{8}$ J. T. Knudtson and E. Weitz, J. Chem. Phys. 83, 927 (1985).

${ }^{9}$ M. A. Bernikov, M. O. Bulanin, and K. G. Tokhadze, J. Mol. Liq. 32, 25 (1986).

${ }^{10}$ M. A. Bernikov, M. O. Bulanin, I. M. Grigoriev, M. V. Tonkov, and K. G. Tokhadze, Opt. Spectrosc. 69, 713 (1990).

${ }^{11}$ M. O. Bulanin, I. M. Grigoriev, M. V. Tonkov, and K. G. Tokhadze, Opt. Spectrosc. 68, 566 (1990).

${ }^{12}$ B. J. van der Veken and F. R. de Munck, J. Chem. Phys. 97, 3060 (1992).

${ }^{13}$ K. G. Tokhadze and Z. Mielke, J. Chem. Phys. 99, 5071 (1993).

${ }^{14}$ K. S. Rutkowski and S. M. Melikova, J. Mol. Struct. 448, 231 (1998).

${ }^{15}$ M. O. Bulanin, K. Kerl, A. Padilla, A. Calvo Hernández, and J. Pérez, Phys. Chem. Chem. Phys. 5, 285 (2003).

${ }^{16}$ A. Padilla, J. Pérez, and A. Calvo Hernández, J. Chem. Phys. 111, 11015 (1999); 111, 11026 (1999).

${ }^{17}$ J. Pérez, D. Luis, A. Calvo Hernández, and S. Velasco, J. Chem. Phys. 91, 3443 (1989).

${ }^{18}$ P. H. Berens and K. R. Wilson, J. Chem. Phys. 74, 4872 (1981).

${ }^{19}$ For a review see, $\mathbf{\square}$, in Molecular Liquids. Dynamics and Interactions, edited by A. J. Barnes, W. J. Orville-Thomas, and J. Yarwood (Reidel, Dordrecht, 1984).

${ }^{20}$ A. Medina, J. M. M. Roco, A. Calvo Hernández, and S. Velasco, J. Chem. Phys. 119, 5176 (2003).

${ }^{21}$ A. Medina, J. M. M. Roco, A. Calvo Hernández, and S. Velasco, Pure Appl. Chem. 76, 241 (2004).

${ }^{22}$ W. F. van Gunsteren and A. E. Mark, J. Chem. Phys. 108, 6109 (1998).

${ }^{23}$ D. Frenkel and B. Smit, Understanding Molecular Simulation (Academic, London, 1996), pp. 379-381. 
${ }^{24}$ F. G. Dijkman and J. H. van der Maas, J. Chem. Phys. 66, 3871 (1977).

${ }^{25}$ S. L. Holmgren, M. Waldman, and W. Klemperer, J. Chem. Phys. 69, 1661 (1978).

${ }^{26}$ J. A. Padró and E. Guàrdia, J. Mol. Liq. 70, 199 (1996).

${ }^{27}$ B. Guillot, Ph. Marteau, and J. Obriot, Mol. Phys. 65, 765 (1988).

${ }^{28}$ K. G. Tokhadze, S. S. Utkina, N. N. Filippov, and Z. Mielke, Opt. Spectrosc. 79, 536 (1995).
${ }^{29}$ J. Pérez, A. Padilla, M. O. Bulanin, A. V. Domanskaya, and K. Kerl, Phys. Chem. Chem. Phys. 6, 725 (2004).

${ }^{30}$ W. A. Herrebout, A. A. Stolov, E. J. Sluyts, and B. J. van der Veken, Chem. Phys. Lett. 295, 223 (1998).

${ }^{31}$ W. Herrebout, B. van der Veken, A. P. Kouzov, and M. O. Bulanin, Phys. Rev. Lett. 92, 023002 (2004). 